\title{
Subinteger Range-Bin Alignment Method for ISAR Imaging of Noncooperative Targets
}

\author{
J. M. Muñoz-Ferreras ${ }^{1}$ and F. Pérez-Martínez ${ }^{2}$ \\ ${ }^{1}$ Department of Signal Theory and Communications, Polytechnic School, University of Alcalá, Campus Universitario, \\ Ctra. Madrid-Barcelona, Km. 33, 600, Alcalá de Henares, 28805 Madrid, Spain \\ ${ }^{2}$ Department of Signals, Systems and Radiocommunications, Technical University of Madrid, E.T.S.I. Telecomunicación, \\ Avenida Complutense s/n, 28040 Madrid, Spain \\ Correspondence should be addressed to J. M. Muñoz-Ferreras,jm.munoz@uah.es
}

Received 17 November 2009; Accepted 25 March 2010

Academic Editor: Robert W. Ives

Copyright ( $\odot 2010$ J. M. Muñoz-Ferreras and F. Pérez-Martínez. This is an open access article distributed under the Creative Commons Attribution License, which permits unrestricted use, distribution, and reproduction in any medium, provided the original work is properly cited.

\begin{abstract}
Inverse Synthetic Aperture Radar (ISAR) is a coherent radar technique capable of generating images of noncooperative targets. ISAR may have better performance in adverse meteorological conditions than traditional imaging sensors. Unfortunately, ISAR images are usually blurred because of the relative motion between radar and target. To improve the quality of ISAR products, motion compensation is necessary. In this context, range-bin alignment is the first step for translational motion compensation. In this paper, we propose a subinteger range-bin alignment method based on envelope correlation and reference profiles. The technique, which makes use of a carefully designed optimization stage, is robust against noise, clutter, target scintillation, and error accumulation. It provides us with very fine translational motion compensation. Comparisons with state-of-the-art rangebin alignment methods are included and advantages of the proposal are highlighted. Simulated and live data from a high-resolution linear-frequency-modulated continuous-wave radar are included to perform the pertinent comparisons.
\end{abstract}

\section{Introduction}

Traditional imaging sensors, such as visible and infrared cameras or laser radar systems, may have a reduced performance in adverse weather conditions, like fog [13]. Furthermore, in defense and security scenarios, smoke screens [4] may literally blind these imaging sensors based on very short wavelengths.

The origin of this degradation must be found in the extreme scattering that these wavelengths suffer when interacting with the little particles present in the atmosphere [13]. When a high signal attenuation is present, the operation range of these sensors diminishes considerably.

However, in important applications related to defense and security, it is still necessary to obtain images for recognition/identification purposes, regardless of the meteorological and scenario conditions.

Inverse Synthetic Aperture Radar (ISAR) is a coherent radar technique which may obtain images of noncooperative targets $[5,6]$. Furthermore, these images may be used for subsequent recognition tasks [7-10]. Although ISAR is usually understood as a complement for electro-optical sensors, it may in fact outperform these traditional sensors in adverse conditions, because it inherits the all-weather feature [11] from the long wavelength radars.

The standard scenario for ISAR consists of a static high-resolution coherent radar which illuminates a moving noncooperative target [12]. In this context, a noncooperative target is a target whose motion is unknown.

In ISAR, the two image dimensions are slant-range and Doppler (cross-range). High slant-range resolution is achieved by transmitting a large bandwidth signal, whereas high cross-range resolution depends on a large aspect angle variation of the target during the illumination time [12]. Specifically, the slant-range resolution is given by

$$
\rho_{r}=\frac{c}{2 \Delta f}
$$


where $c$ is the light speed and $\Delta f$ is the transmitted bandwidth. The cross-range resolution may be written as

$$
\rho_{a}=\frac{\lambda}{2 \Delta \theta}
$$

where $\lambda$ is the transmitted wavelength and $\Delta \theta$ is the variation of the target aspect angle during the illumination (observation) time.

Target motion may be divided into a translational component and a rotational component $[13,14]$. The first one is further decomposed into a radial and a tangential component, whereas the second one has three attitude components: yaw, pitch, and roll.

On the one hand, the radial component of the translational motion (i.e., the component along the line-of-sight (LOS)) is undesired, because it does not induce variation of the target aspect angle; that is, it does not generate Doppler gradient among target scatterers situated in the same range bin. Furthermore, this component causes a large blurring in ISAR images.

On the other hand, the rest of motion components may produce the desired Doppler gradient among scatterers, hence obtaining bidimensional information. It is true that the rotational motion (and the tangential component of the translational motion) may also generate blurring effects on the image [15], called Migration Through Resolution Cells (MTRCs), but these effects have minor importance in comparison to the large blurring generated by the radial component of the translational motion, which must always be compensated.

Methods for translational motion compensation work in two steps [16]: range-bin alignment and phase adjustment. For the first stage, which is the motivation of this paper, several methods may be found in the literature, such as the peak tracking approach [5], the centroid tracking algorithm [17], the envelope correlation method $[5,18,19]$, the global range alignment approach [20], or the minimum-entropybased technique [21]. For the second stage regarding phase adjustment, the literature provides us with famous methods such as prominent point processing [22], phase gradient autofocus [23], entropy minimization [16], or contrast maximization [24].

In this paper, we concentrate on the range-bin alignment stage, which is fundamental to guarantee a proper translational motion compensation. Concretely, we present a subinteger range-bin alignment approach based on the traditional envelope correlation method and the use of reference profiles. This work was preliminarily presented in the conference paper [19]. Here, deep analyses as well as exhaustive comparisons with other existing methods both for simulated and live data are provided.

The proposed method makes use of reference profiles in order to mitigate the error accumulation phenomenon and the target scintillation effects [18], typical limitations of the earlier range-bin alignment approaches such as the peak and centroid tracking methods. Furthermore, the technique makes a subinteger alignment which provides us with a very fine range profile adjustment. This subinteger

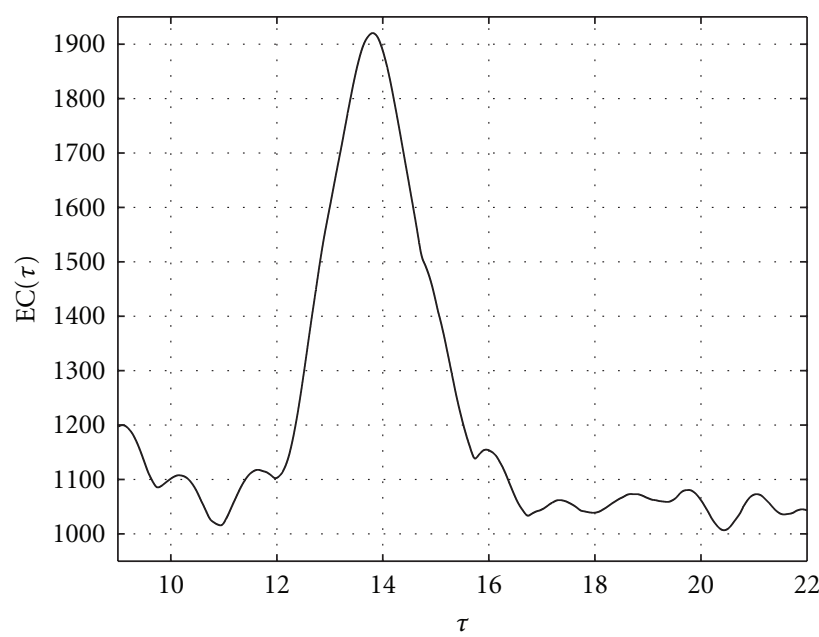

FIGURE 1: Envelope correlation (as a function of the range shift $\tau$ ) between a real shifted range profile and its corresponding reference profile.

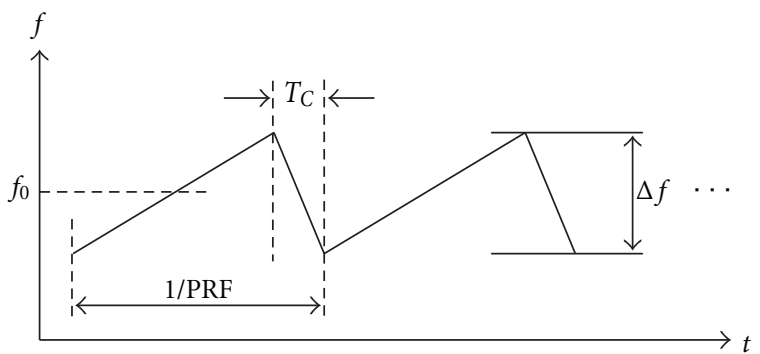

Figure 2: Waveform for an LFMCW radar.

alignment approach is based on an optimization stage which has carefully been designed in order to avoid possible convergence to local maxima.

The method is robust against noise, clutter, and target scintillation. Moreover, it properly solves the error accumulation problem. Its performance is similar to the state-ofthe-art current methods such as the global range alignment approach [20] and the minimum entropy-based technique [21], although it provides two clear advantages; it properly solves extreme situations with large range shifts from pulse to pulse (unlike the global range alignment algorithm) and it has moreover the ability to produce subinteger fine range adjustments over a wide range of offsets (in contrast to the minimum entropy-based method).

Furthermore, the careful design of the method against local maxima makes it very robust, as shown here for controlled simulated examples for which the state-of-the-art methods have convergence problems.

Simulated and live data from a high-resolution linearfrequency-modulated continuous-wave (LFMCW) radar are used to validate the proposed approach and to make the pertinent comparisons. 


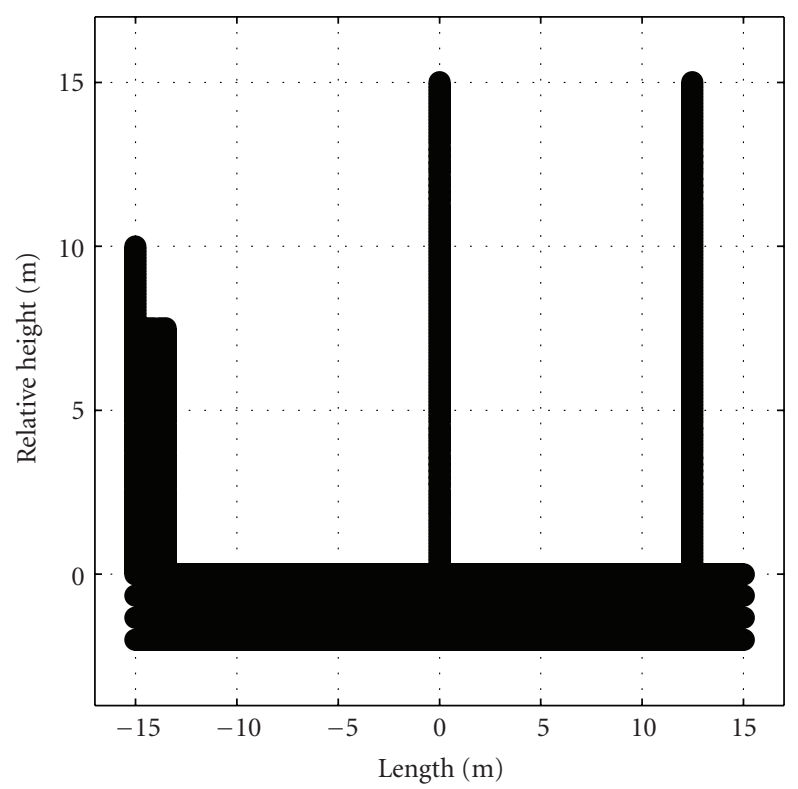

FIGURE 3: Scatterer distribution for a simulated target illuminated by an LFMCW radar.

\section{Subinteger Range-Bin Alignment Method}

The proposed method uses the cross-correlation of range profiles in order to estimate the misalignment between them. The correlation is not calculated between the current range profile and the previous one, which would generate the undesired error accumulation effect [18]. On the contrary, the cross-correlation is calculated between the current range profile and a reference profile obtained as a combination of the previously aligned range profiles. This reduces the error accumulation effect and provides robustness against noise, clutter, and target scintillation. Moreover, the proposed alignment between the current and the reference profiles may be a fraction of one range bin, providing the method with a subinteger capability. This fine alignment is achieved after an optimization stage which has been designed to minimize possible convergence to local maxima. The following paragraphs describe the method.

Let $\mathbf{p}_{m}(n)$ be the $m$ th acquired range profile, where $n=$ $0, \ldots, N-1, m=0, \ldots, M-1, N$ is the total number of range bins and $M$ is the number of acquired range profiles. Let us call $\overline{\mathbf{p}}_{m}(n)$ as the aligned profile of $\mathbf{p}_{m}(n)$, after the alignment process.

As previously commented, in order to reduce the error accumulation effect and to increase the method robustness against noise, clutter, and target scintillation, it is interesting to define a reference profile [18]. We calculate this reference profile as a combination of the previously aligned range profiles. Concretely, we follow the recommendations of [16] in order to define the reference profile $\mathbf{r}_{m+1}(n)$ for the alignment of $\mathbf{p}_{m+1}(n)$ as

$$
\mathbf{r}_{m+1}(n)=\frac{m}{m+1} \mathbf{r}_{m}(n)+\frac{1}{m+1}\left|\overline{\mathbf{p}}_{m}(n)\right|
$$

where $\mathbf{r}_{m}(n)$ is the reference profile for the alignment of the $m$ th range profile $\mathbf{p}_{m}(n)$. Note that the calculation of $\mathbf{r}_{m+1}(n)$ requires the knowledge of the previously aligned range profiles. This clearly demonstrates the iterative nature of the method.

Once the $(m+1)$ th range profile $\mathbf{p}_{m+1}(n)$ and its associated reference profile $\mathbf{r}_{m+1}(n)$ are available, the objective is to obtain the $(m+1)$ th-aligned range profile $\overline{\mathbf{p}}_{m+1}(n)$. For this purpose, the envelope cross-correlation between $\mathbf{r}_{m+1}(n)$ and a shifted version of $\mathbf{p}_{m+1}(n)$ is defined as

$$
\operatorname{EC}\left(\tau_{m+1}\right)=\sum_{n=0}^{N-1}\left|\mathbf{r}_{m+1}(n)\right| \cdot\left|\mathbf{p}_{m+1}\left(n-\tau_{m+1}\right)\right|,
$$

where $\tau_{m+1}$ is the range shift applied to the $(m+1)$ th range profile $\mathbf{p}_{m+1}(n)$. The value $\tau_{m+1}$ is not necessarily an integer, so that $\mathbf{p}_{m+1}\left(n-\tau_{m+1}\right)$ is calculated by using the shift property of the Fourier transform as follows:

$$
\mathbf{p}_{m+1}\left(n-\tau_{m+1}\right)=\operatorname{FFT}\left\{e^{j(2 \pi / N) \tau_{m+1} \mathbf{n}} \operatorname{IFFT}\left\{\mathbf{p}_{m+1}(n)\right\}\right\},
$$

where $\mathbf{n}$ is the vector $[0,1, \ldots, N-1]^{T}$.

In the context of this paper, a maximum value of the envelope correlation is indicative of an optimum alignment between the $(m+1)$ th reference profile $\mathbf{r}_{m+1}(n)$ and the shifted range profile $\mathbf{p}_{m+1}\left(n-\tau_{m+1}\right)$. Hence, we are interested in obtaining the optimum shift $\hat{\tau}_{m+1}$ that maximizes the envelope correlation. Mathematically, this optimum shift may be expressed as

$$
\hat{\tau}_{m+1}=\arg \max _{\tau_{m+1}} \operatorname{EC}\left(\tau_{m+1}\right) .
$$

After solving the optimization problem, we can finally obtain the aligned profile $\overline{\mathbf{p}}_{m+1}(n)$ as

$$
\overline{\mathbf{p}}_{m+1}(n)=\mathbf{p}_{m+1}\left(n-\hat{\tau}_{m+1}\right),
$$

where the range shift of the original profile is implemented by using (5), because this optimum shift $\hat{\tau}_{m+1}$ may be not an integer.

It is really interesting to analyze the optimization problem expressed in (6) in order to visualize its nature. Figure 1 shows the value of the envelope correlation (see (4)) between a live-shifted range profile and its associated reference profile as a function of the shift $\tau$. In this example, the variable $\tau$ is expressed in number of range bin.

The envelope correlation shown in Figure 1 shows a standard example which we have found typical for both simulated and real data. It can easily be seen that the objective cost function suffers from local maxima.

As a consequence, it is obvious that we have to pay great attention to the correct initialization of the optimization algorithm in order to guarantee the desired convergence to the global maximum. That is, if the initial guess for the optimization method is near to the global maximum, a correct convergence to it is achievable.

Since the problem under study is an optimization problem with local maxima, it is clear that we could employ blind approaches to solve it, such as genetic algorithms or 


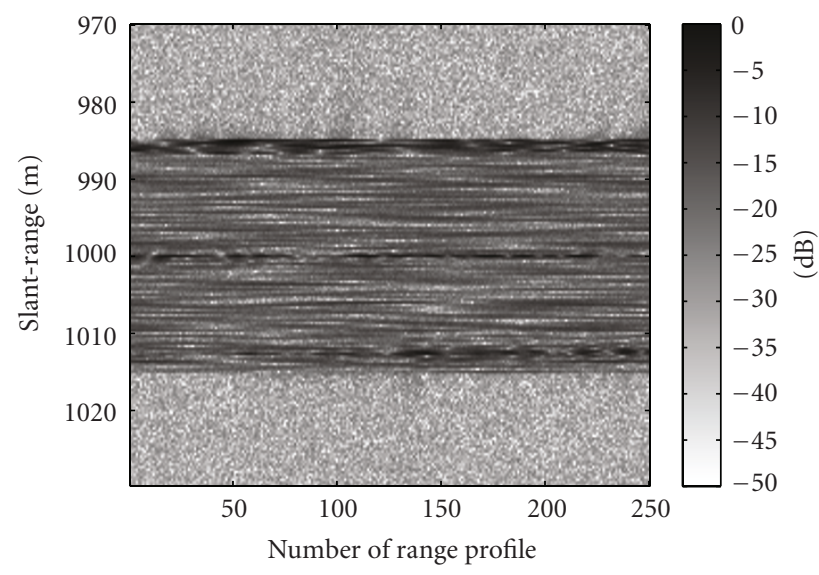

(a)

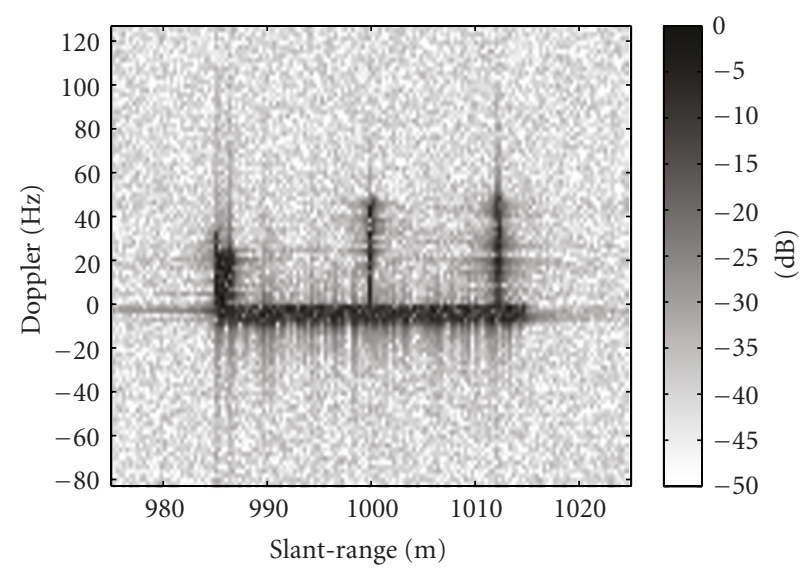

(b)

FIGURE 4: (a) Range profiles and (b) ISAR image for the simulated example without radial translational velocity.

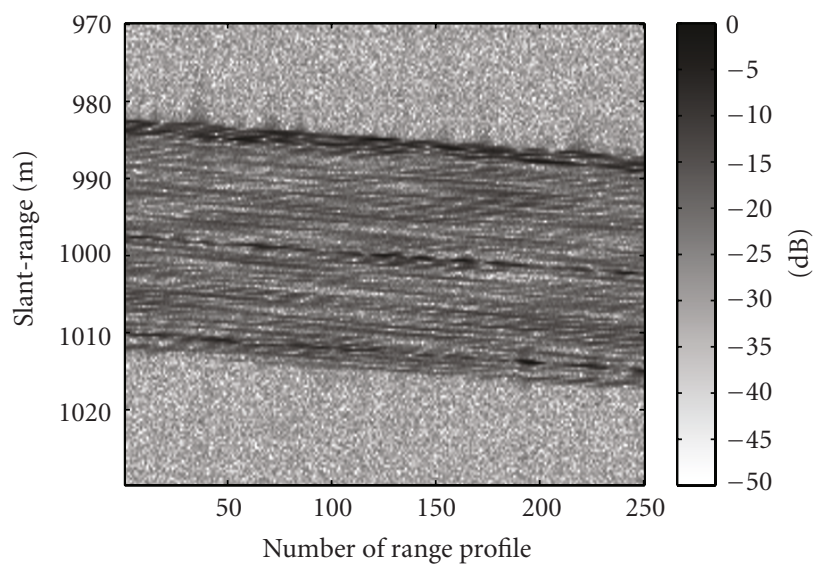

(a)

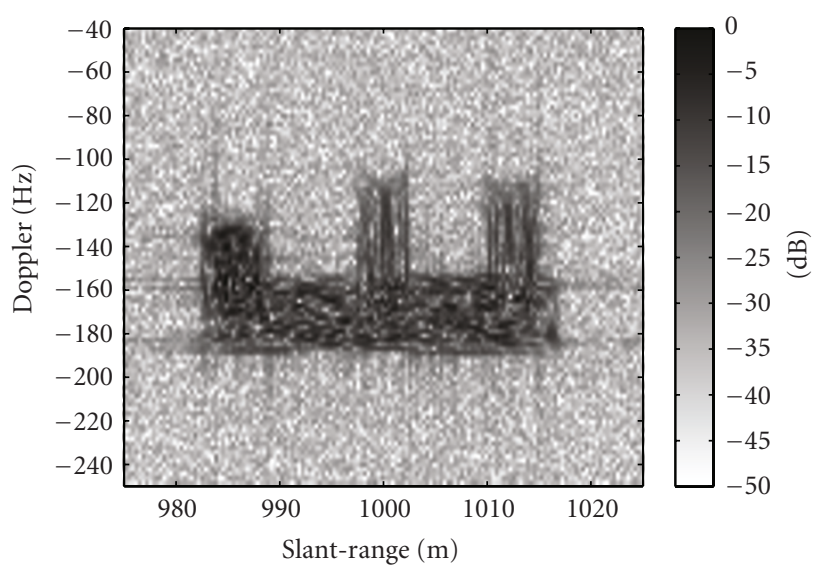

(b)

FIGURE 5: (a) Range profiles and (b) ISAR image for the simulated example with a radial translational velocity of $v_{r}=10 \mathrm{~m} / \mathrm{s}$.

exhaustive procedures (like a grid method or a random walk).

Nevertheless, according to our observations of our available simulated and real data, we have noticed that the peak corresponding to the global maximum of the cost function is quite wide, as seen in Figure 1. This means that a proper initial guess for the optimum shift $\hat{\tau}_{m+1}$ is simply the integer range shift for which the cross-correlation is maximum. Hence, we evaluate the cross-correlation for the possible integer shifts $n=0, \ldots, N-1$, and select the one for which the cost function is maximum as the initial guess for the subsequent standard optimization algorithm. This process lets us finally converge to the desired global maximum. In this paper, a zero-order optimization algorithm (the NelderMead algorithm [25]) has been utilized to obtain the desired subinteger refinement for $\hat{\tau}_{m+1}$.

We would like to highlight that the commented method to obtain the initial guess properly worked in all the simulated and real data available to the authors. On the other hand, it is true that other optimization approaches, like a gradient-based method or the Newton method, may have been used for the refinement stage. Our experience is that the zero-order Nelder-Mead algorithm worked properly for the analyzed examples.

As a summary, the following steps implement the proposed technique for ISAR subinteger range-bin alignment.

Step $1(m=0)$. Consider $\overline{\mathbf{p}}_{0}(n)=\mathbf{p}_{0}(n)$.

Step 2. Calculate the $(m+1)$ th reference profile $\mathbf{r}_{m+1}(n)$ using (3).

Step 3. Obtain the envelope correlation (see (4)) between $\mathbf{r}_{m+1}(n)$ and the $(m+1)$ th-shifted range profile $\mathbf{p}_{m+1}(n-$ $\left.\tau_{m+1}\right)$, with $\tau_{m+1}$ being an integer in the interval $[0,1, \ldots, N-$ $1]$ and $N$ the number of range bins.

Step 4. Calculate the integer value of $\tau_{m+1}$, from the possible set $[0,1, \ldots, N-1]$, that maximizes the result of the previous step. Call this initial value $\tau_{m+1,0}$. 


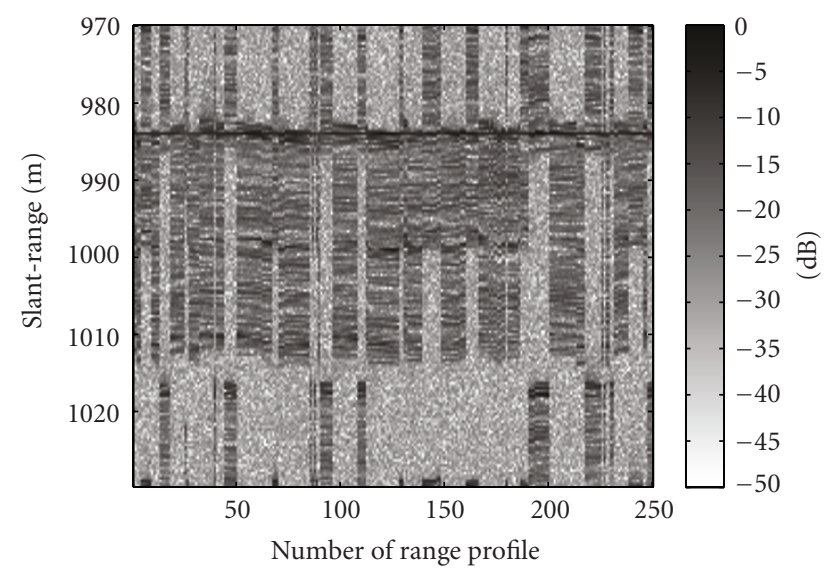

Figure 6: Range profiles for the simulated example after applying the peak tracking method. Note that the target scintillation phenomenon makes this approach fail.

Step 5. Solve (6) with the Nelder-Mead algorithm, taking $\tau_{m+1,0}$ as the initial guess for the iterative technique. As a result, obtain the optimum range shift $\hat{\tau}_{m+1}$.

Step 6. Obtain $\overline{\mathbf{p}}_{m+1}(n)$ using (7). Use (5) if the optimum shift $\hat{\tau}_{m+1}$ is not an integer.

Step $7(m \leftarrow m+1)$. If $m \leq M-2$, where $M$ is number of range profiles, go to Step 2 to align the next range profile.

\section{Simulation of an LFMCW Radar}

In this section, we provide the tools to simulate targets illuminated by an LFMCW radar, because the simulated and real data used in this paper correspond to this type of radar. Next section will detail the properties of the proposed alignment method for this kind of data. Although the results detailed in the paper only refer to LFMCW radars, it is important to highlight that the proposed method is applicable to any coherent imaging radar.

An LFMCW radar transmits a continuous waveform whose instantaneous frequency as a function of the time is depicted in Figure 2. The central transmitted frequency is $f_{0}$, the pulse repetition frequency is PRF, the transmitted bandwidth is represented by $\Delta f$, whereas the parameter $T_{C}$ represents the necessary time for the radar circuits to guarantee the signal coherence from ramp to ramp.

Next, we describe the radar signal model. The complex envelope of the transmitted signal $s_{T}(t)$ may be written as

$$
s_{T}(t)=\exp \left(j\left(2 \pi f_{0} t+\pi \gamma \tilde{t}^{2}\right)\right)
$$

where $\tilde{t}=t-m \mathrm{PRF}^{-1}, t$ is the time, $m$ is the number of range profile (i.e., the number of ramp), and $\gamma$ is the chirp rate. Note that the received signal in the intervals corresponding to $T_{C}$ is not processed.
TABLE 1: LFMCW radar parameters for the simulated example in Figure 3.

\begin{tabular}{lc}
\hline Central frequency $\left(f_{0}\right)$ & $10 \mathrm{GHz}$ \\
Bandwidth $(\Delta f)$ & $500 \mathrm{MHz}$ \\
Ramp Repetition Frequency $(\mathrm{PRF})$ & $500 \mathrm{~Hz}$ \\
$T_{C}$ & $0.2 \mathrm{~ms}$ \\
Illumination Time $(\mathrm{CPI})$ & $0.5 \mathrm{~s}$ \\
\hline
\end{tabular}

If we consider a point-scatterer at a range $R_{t k}$ from the radar, the received signal from the scatterer is

$$
s_{R k}(t)=\sigma_{k} \exp \left(j\left(2 \pi f_{0}\left(t-\frac{2 R_{t k}}{c}\right)+\pi \gamma\left(\tilde{t}-\frac{2 R_{t k}}{c}\right)^{2}\right)\right),
$$

where $\sigma_{k}$ is a complex value associated to the scatterer, whose amplitude represents the scatterer backscattering and the propagation losses, whereas its phase models a possible phase change inserted by the scatterer.

LFMCW radars usually apply hardware deramping, which consists of mixing the received signal with a replica of the transmitted signal. By considering that the target has $K$ scatterers, the beat signal after the deramping processing may be written as

$$
s_{b}(t)=\sum_{k=1}^{K} \sigma_{k} \exp \left(j\left(\frac{4 \pi \gamma R_{t k}}{c} \tilde{t}+\frac{4 \pi f_{0}}{c} R_{t k}-\frac{4 \pi \gamma R_{t k}^{2}}{c^{2}}\right)\right) .
$$

Note that, for each transmitted ramp, a range profile may be obtained. For an LFMCW radar, it is necessary to make a Fourier transform of the beat signal to obtain the range profiles. Effectively, by neglecting the last phase term in (10), it is clear that a Fourier transform in $\tilde{t}$ supplies the range profile for each $m$. The beat frequency $f_{t k}$ for a scatterer situated at a range $R_{t k}$ may be written, from (10), as

$$
f_{t k}=\frac{2 \gamma R_{t k}}{c} .
$$

A correct sampling of (10) provides us with the possibility of simulating complex scenes. This is simply made by calculating the ranges from the radar to all target scatterers for all ramps. For each ramp, the corresponding range profile may be obtained by applying a Fourier transform to (10).

As an interesting example, let us consider the distribution of 2000 scatterers depicted in Figure 3. Let us say that these scatterers belong to a ship.

Let us consider that this target, while pitching with a rotation rate of $\Omega=0.05 \mathrm{rad} / \mathrm{s}$, is moving away along LOS and is being illuminated by an LFMCW radar. The radar parameters are detailed in Table 1, where the illumination time is also referred as Coherent Processing Interval (CPI). The rotation center of the target is situated in the position $[0,0]$ in Figure 3. Moreover, let us consider that the range from the radar to the rotation center is $1000 \mathrm{~m}$ in the middle of the illumination time. 


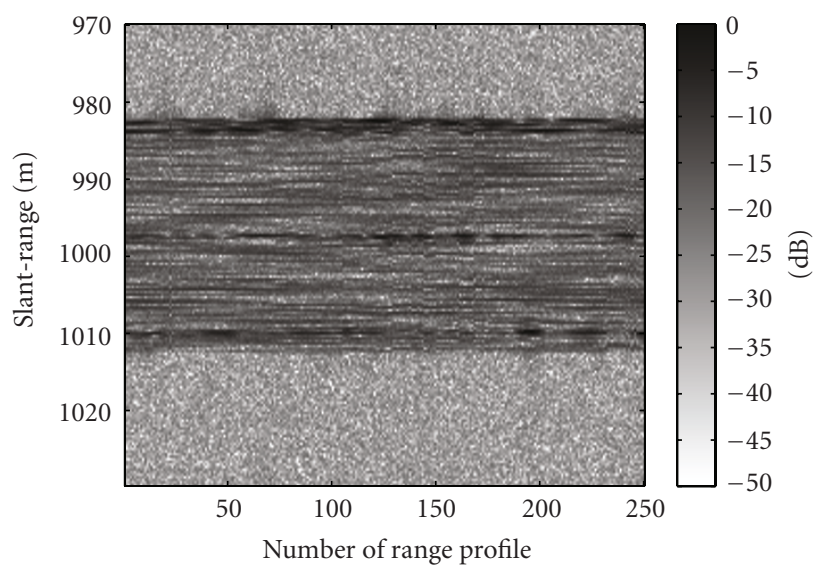

(a)

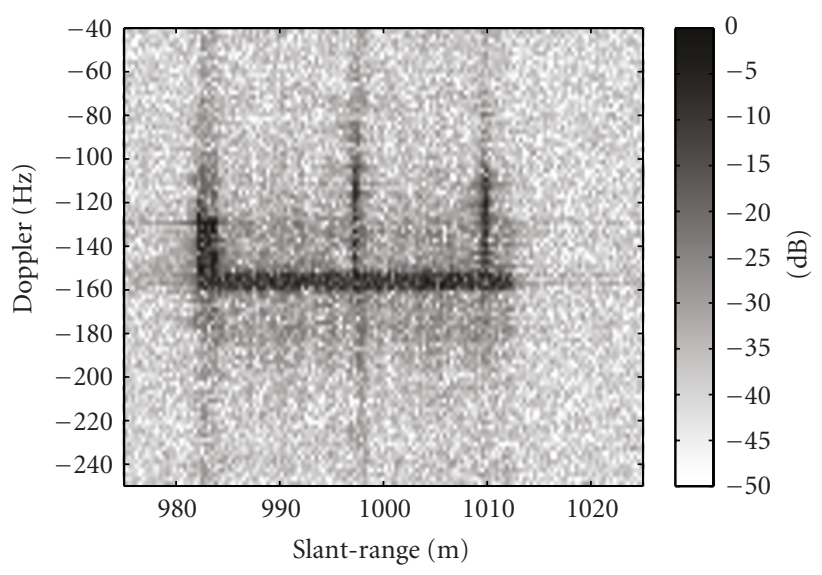

(b)

FIGURE 7: (a) Range profiles and (b) reconstructed ISAR image after applying the proposed subinteger range-bin alignment method to the simulated data with translational motion.

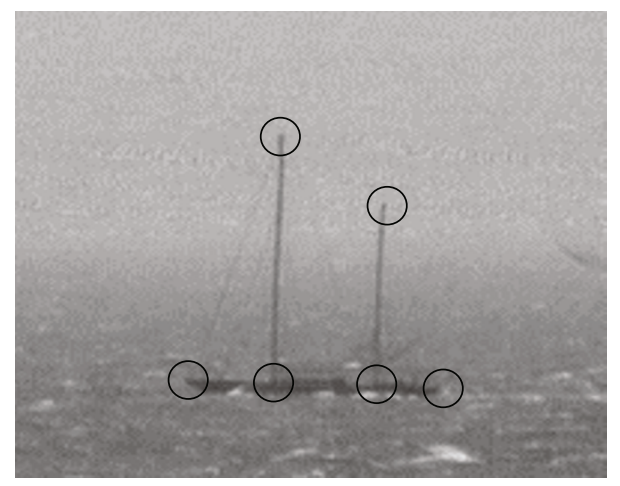

FIgURE 8: Photo of the two-mast sailboat.

For this simulated example, we have considered that $\left|\sigma_{k}\right|=1$, for all $k$ and $\angle \sigma_{k}$ is uniformly distributed between 0 and $2 \pi$. Moreover, a signal-to-noise ratio of $10 \mathrm{~dB}$ has been considered, with the noise being additive, white, and Gaussian.

Figure 4(a) shows the 250 range profiles for this example, when the target is considered to have a radial translational speed of $v_{r}=0 \mathrm{~m} / \mathrm{s}$. By applying an FFT in each range bin, we obtain the ISAR image in the conventional rangeDoppler coordinates (Figure 4(b)). In the context of this paper, Figure 4(b) must be considered as the optimum ISAR image for this simulation, because there is no blurring (i.e., the radial component of the translational motion is zero).

On the other hand, Figures 5(a) and 5(b), respectively, show the range profiles and the ISAR image for the previously simulated example, but considering that the target is moving away with a radial speed of $v_{r}=10 \mathrm{~m} / \mathrm{s}$. The leaning observed in the range profiles and the large blurring in the ISAR image are characteristic effects due to the radial component of the translational motion.

Because the dynamics for noncooperative targets are unknown, the objective of the blind motion compensation techniques consists of focusing the ISAR images without additional information. For our simulated example, the blind techniques should obtain an image similar to Figure 4(b) from processing the data in Figure 5.

Next sections detail the performance of the proposed alignment algorithm in comparison with other existing methods for compensating the translational motion. In order to make fair comparisons among the diverse rangebin alignment techniques, in this paper we always use the method in [16] for the phase adjustment step.

\section{Properties of the Proposed Method}

This section addresses the performance of the proposed range-bin alignment technique in relation to important features: robustness against target scintillation, against clutter, and so forth. Both simulated and real data are used to verify the good performance of the proposed method.

4.1. Robustness against Target Scintillation. The signal received by the radar is the coherent sum of many contributions from target scatterers. This implies that the power in each range bin is not constant during the illumination time. This effect is known as target scintillation.

Target scintillation makes the standard tracking approaches fail. For example, Figure 6 shows the range profiles for our simulated example with a radial translational motion aligned after applying the peak tracking method. Clearly, the simulated data suffer from the target scintillation phenomenon, because we have simulated many pointscatterers. Target scintillation causes the location of the global maximum to strongly fluctuate between range profiles.

Fortunately, the subinteger range-bin alignment method does not suffer from target scintillation. Note that envelope correlation is a much more robust approach to calculate the existing shift among range profiles. As a proof of it, Figure 7 (a) details the range profiles obtained after applying the proposed method to the simulated data of Figure 5. It can 


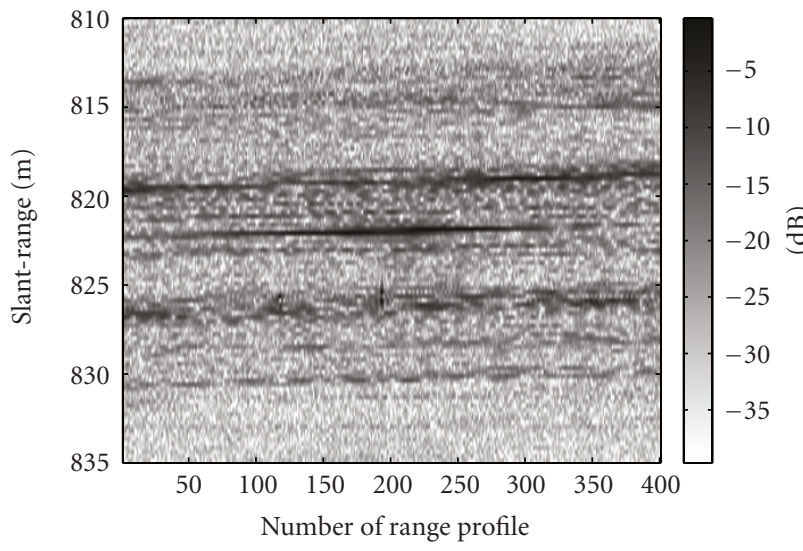

(a)

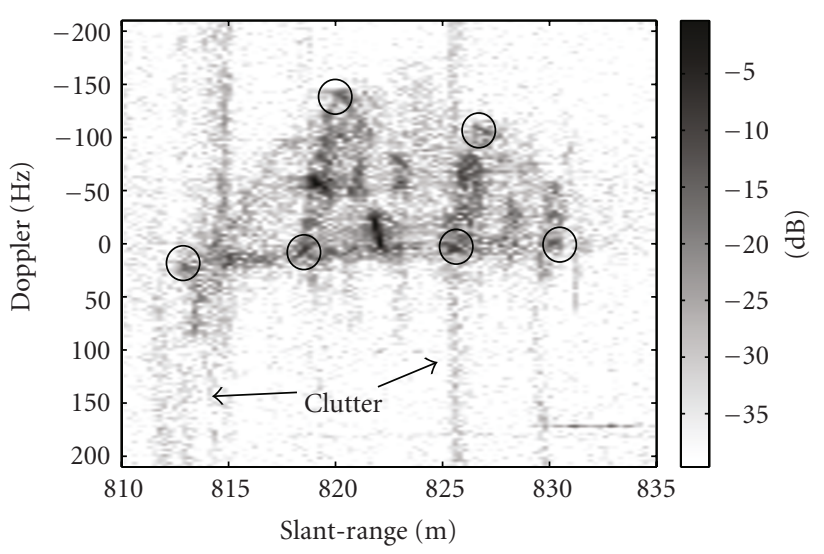

(b)

FIgURE 9: (a) Range profiles and (b) ISAR image for the real data of the two-mast sailboat. No motion compensation technique has been applied.

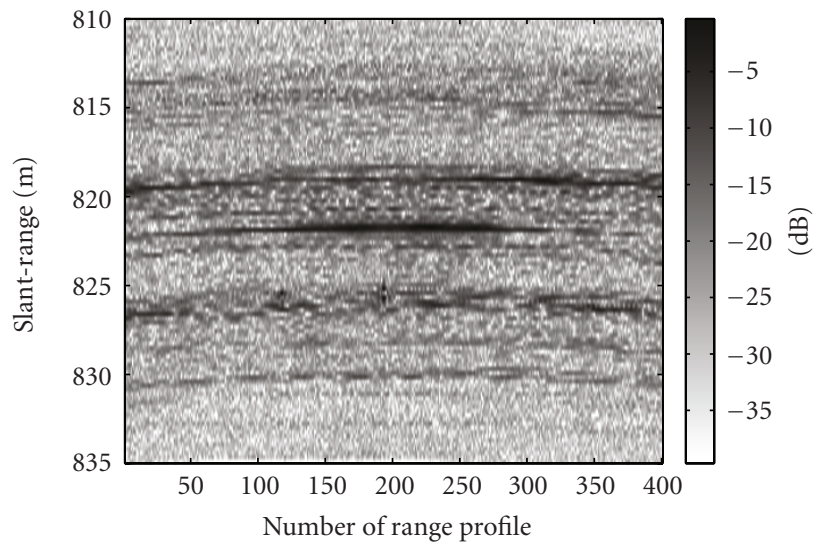

(a)

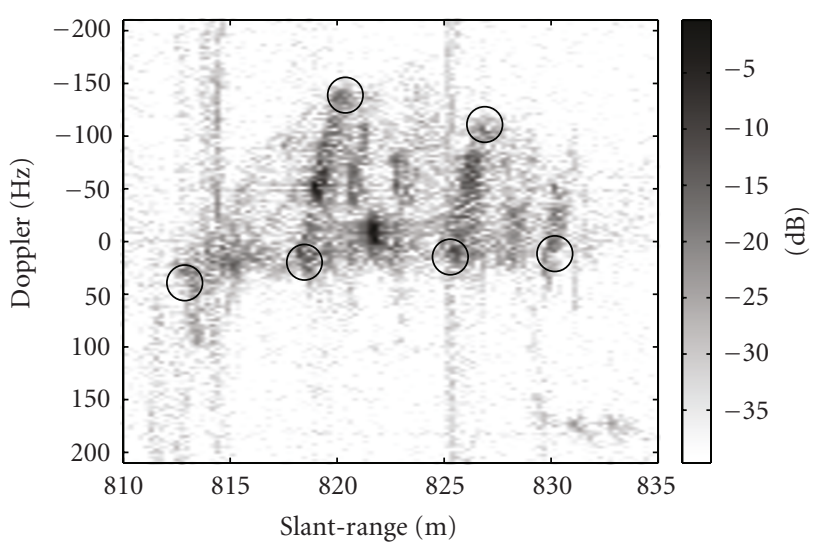

(b)

FIGURE 10: (a) Range profiles and (b) ISAR image for the real data of the two-mast sailboat after applying the centroid tracking method.

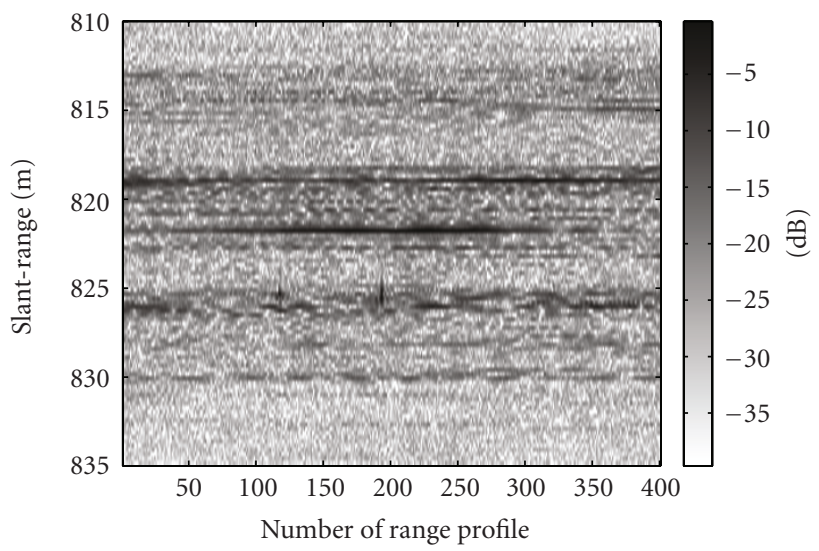

(a)

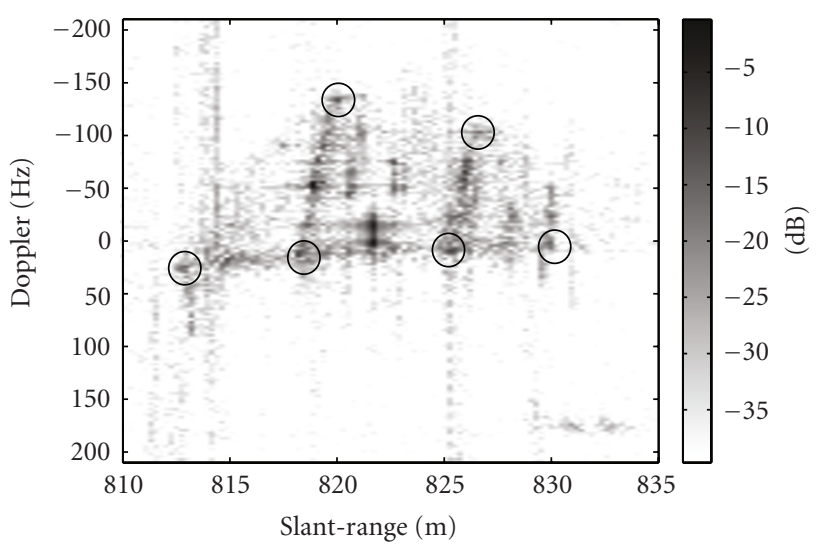

(b)

FIGURE 11: (a) Range profiles and (b) ISAR image for the real data of the two-mast sailboat after applying the proposed method. 


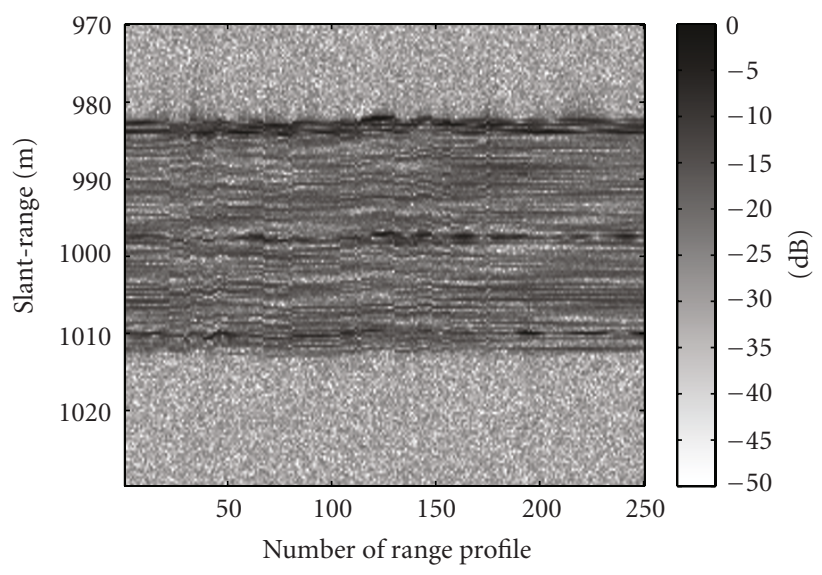

(a)

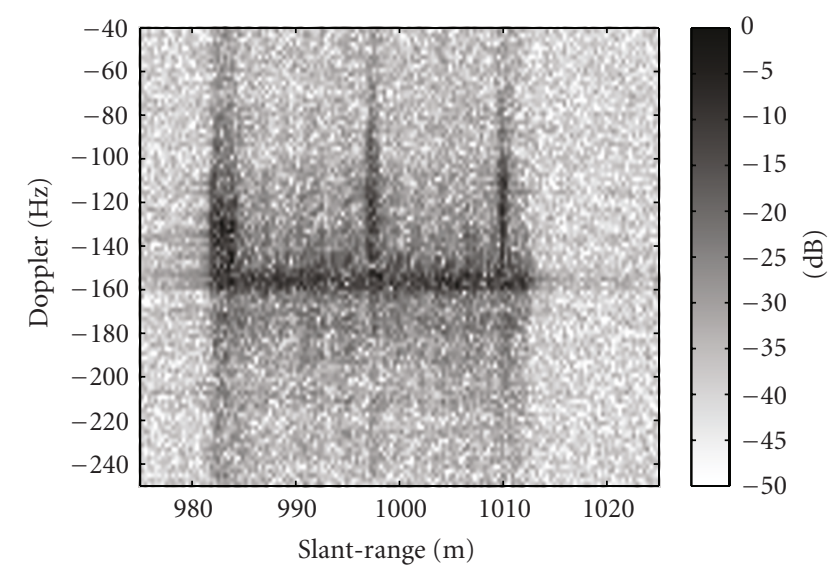

(b)

FIgURE 12: (a) Range profiles and (b) ISAR image without using reference profiles. The results correspond to the simulated data presented in Figure 5.

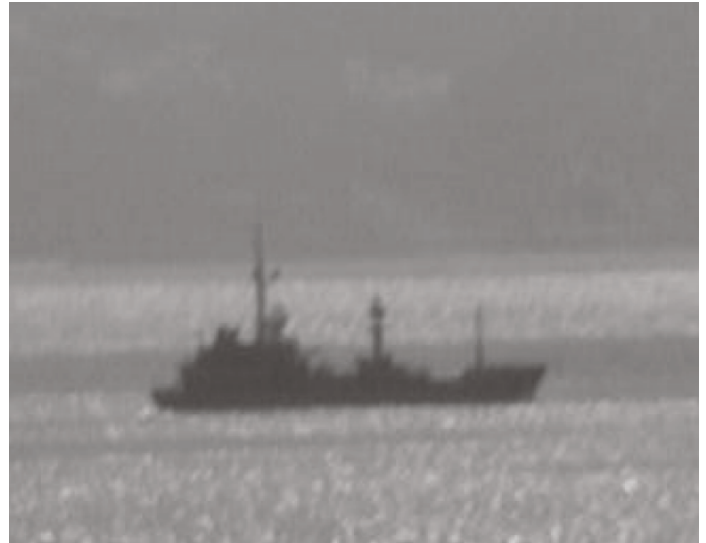

Figure 13: Photo of the vessel.

be seen that a proper range-bin alignment has been obtained. In fact, this result is very similar to the range profiles shown in Figure 4(a), which may be understood as the optimum range profiles, because they correspond to the simulated example without translational motion.

Figure 7(b) shows the motion-compensated ISAR image obtained with the proposed technique for the range-bin alignment stage and with the method in [16] for the phase adjustment stage. Hence, Figure 7 (b) is the reconstructed ISAR image after using the proposed technique. Note that this reconstruction is a very good approximation to the optimum ISAR image shown in Figure 4(b).

4.2. Robustness against Noise and Clutter. Thermal noise is always present in real systems. The simulated examples shown in this paper include thermal noise. On the other hand, clutter may exist depending on the acquisition scenario. For example, in maritime scenarios, the clutter due to echoes from the sea may be a problem.

Figure 8 shows the photograph of a noncooperative twomast sailboat, which was illuminated during an acquisition
TABLE 2: Real radar parameters for the acquired data corresponding to the sailboat in Figure 8.

\begin{tabular}{lc}
\hline Central frequency $\left(f_{0}\right)$ & $28.5 \mathrm{GHz}$ \\
Bandwidth $(\Delta f)$ & $1 \mathrm{GHz}$ \\
Ramp repetition frequency $(\mathrm{PRF})$ & $1000 \mathrm{~Hz}$ \\
$T_{C}$ & $0.1 \mathrm{~ms}$ \\
Illumination time $(\mathrm{CPI})$ & $0.4 \mathrm{~s}$ \\
\hline
\end{tabular}

campaign made in the Strait of Gibraltar. The circles approximately indicate the positions of the mast bases and tips and the sailboat bow and stern. The real radar [26] is a high-resolution millimeter-wave LFMCW radar, whose parameters for this acquisition are detailed in Table 2.

This live example is an interesting one, since the sea state was 4 and many clutter echoes were received. Figures 9(a) and 9(b), respectively, show the range profiles and the ISAR image for this capture without applying any motion compensation technique. We can see that the signal-to-noise ratio is poor and that energetic echoes corresponding to clutter are evident. Moreover, the ISAR image is blurred because of the translational motion of the target, which is evident from the characteristic leaning observed in the range profiles in Figure 9(a). Certainly, it is difficult to distinguish the details of the ship in the ISAR image shown in Figure 9(b).

The high levels of clutter and noise have influence on the performance of the standard tracking-based techniques. As an example, refer to the range profiles aligned after applying the centroid tracking-based technique [17], which are detailed in Figure 10(a). Clearly, by simple visual inspection, we can conclude that the alignment has not been satisfactory. Figure 10(b) shows the ISAR image obtained after applying the centroid tracking method for the range-bin alignment stage (and the method in [16] for the phase adjustment stage). The quality of the ISAR image has not improved in relation to Figure 9(b), that is, the ISAR image is still blurred. 


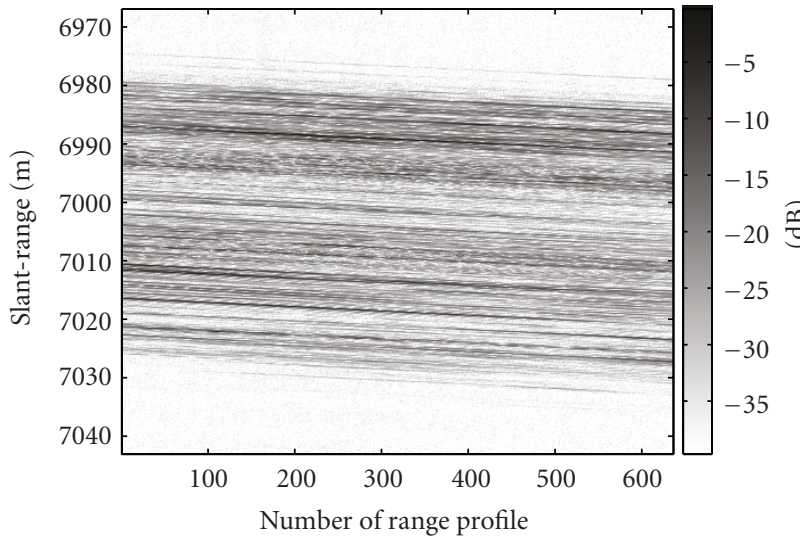

(a)

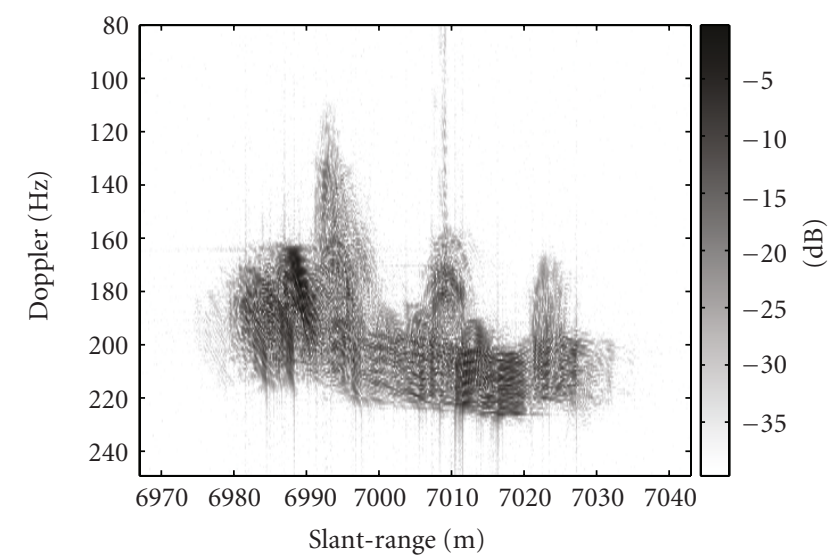

(b)

FIGURE 14: (a) Range profiles and (b) ISAR image for the real data of the vessel without compensating the translational motion.

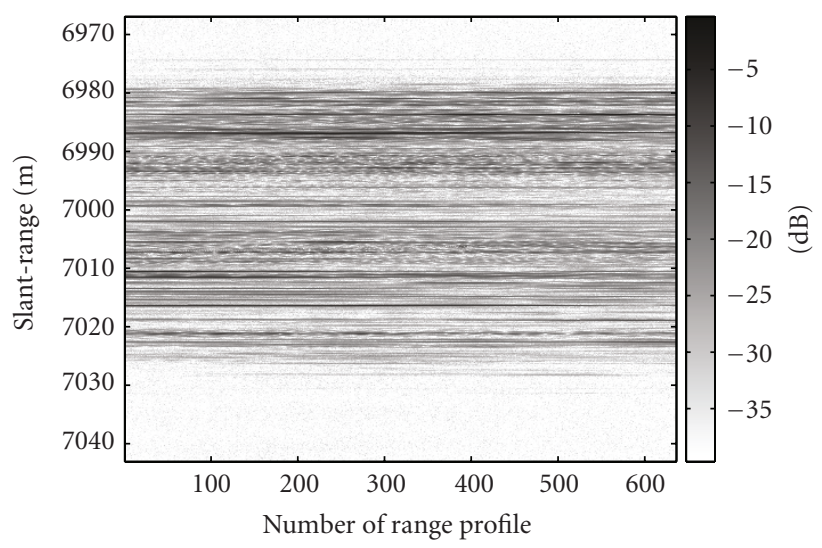

(a)

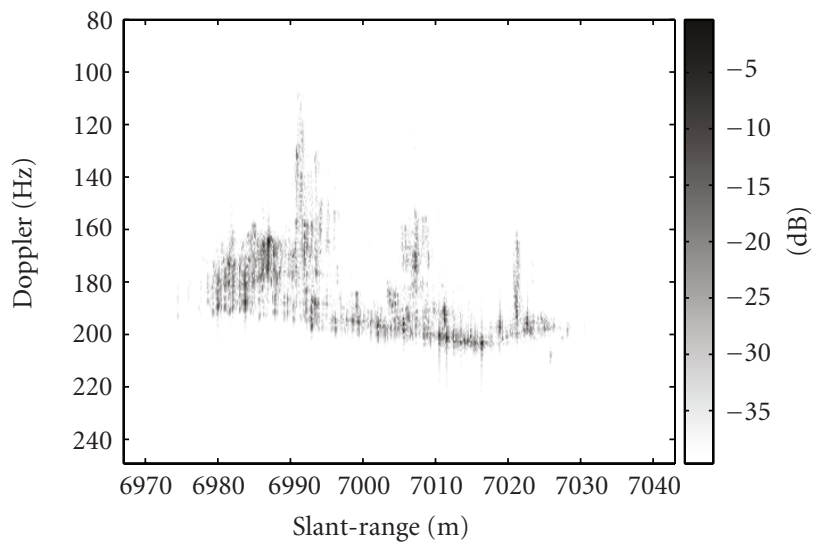

(b)

FIGURE 15: (a) Range profiles and (b) ISAR image for the real data of the vessel after applying the proposed method.

Certainly, the high levels of noise and clutter for this example make the tracking-based methods fail.

On the other hand, Figure 11(a) presents the range profiles aligned by the proposed subinteger range-bin alignment method. In this case, we can see that the range profiles are more properly aligned. Figure 11(b) shows the reconstructed ISAR image for this case, where the masts and the deck are more detailed in comparison to Figures 9(b) and 10(b).

Hence, the proposed range-bin alignment method is robust against high levels of noise and clutter. So far, the drawn conclusions are based on visual inspection. However, we can use focusing indicators to quantify the quality improvement observed in the ISAR image of Figure 11(b). In this context and for this kind of examples, we can use the entropy [16] and the contrast [24], whose mathematical definitions are given in the corresponding references. The lower the entropy, the more focused the ISAR image is [16]. And, the greater the contrast, the more focused the ISAR image is [24].

These focusing indicators for the ISAR images of Figures 9(b), 10(b), and 11(b) are detailed in Table 3. According to these results, we have that the ISAR image after applying the
TABLE 3: Focusing indicators for the live ISAR images in Figures 9(b), 10(b), and 11(b).

\begin{tabular}{lcc}
\hline & Entropy & Contrast \\
\hline Figure 9(b) & 7.34 & 17.41 \\
Figure 10(b) & 7.46 & 15.90 \\
Figure 11(b) & 6.90 & 24.80 \\
\hline
\end{tabular}

proposed technique is more focused than the original image without compensating the translational motion and is also more focused than the image obtained with the trackingbased range-bin alignment approach. We even obtain that the quality of the ISAR image after applying the centroid tracking technique gets worse in relation to Figure 9(b).

4.3. Robustness against Error Accumulation. The error accumulation phenomenon is an effect observed when using some range-bin alignment methods [18]. The proposed method tries to mitigate this phenomenon by using reference profiles, whose calculation is based on the previously aligned range profiles. 


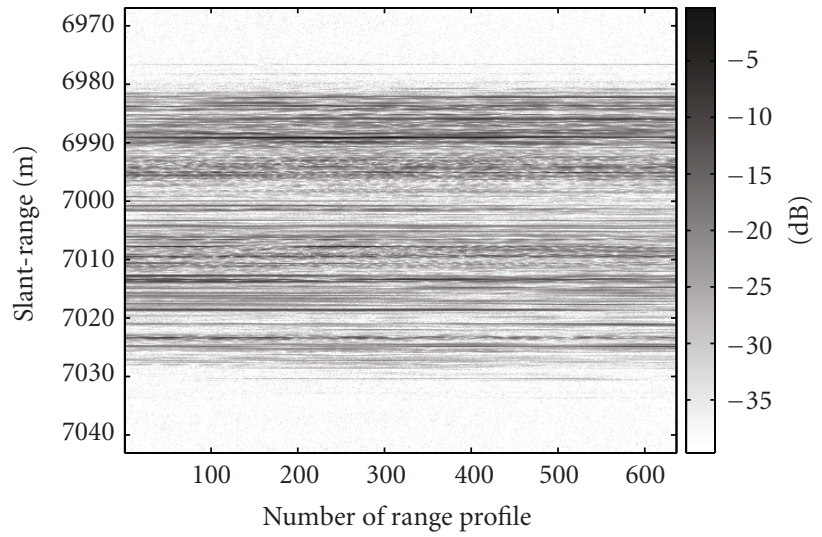

(a)

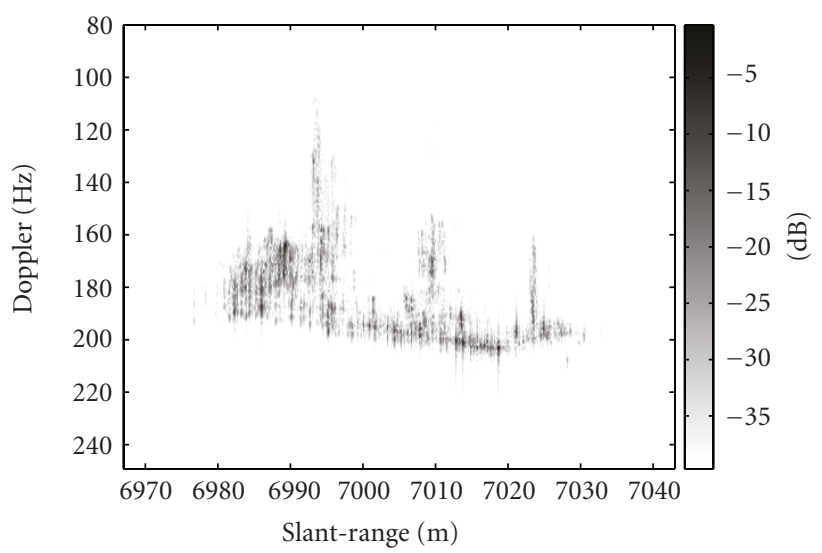

(b)

FIGURE 16: (a) Range profiles and (b) ISAR image for the real data of the vessel after using the global range alignment algorithm.

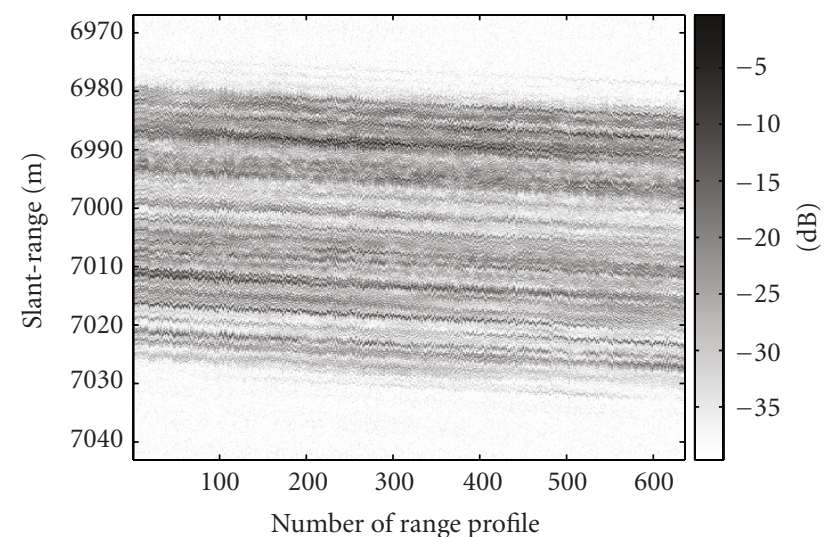

FIGURE 17: Range profiles of the vessel with an artificially induced vibration.

For example, for envelope correlation-based methods, the error accumulation effect takes importance when the alignment of the current range profile is only based on the previously aligned range profile, as shown next.

Figure 12(a) shows the range profiles obtained after using a reduced version [5] of the proposed method for aligning the range profiles of the simulated example in Figure 5. This simplified algorithm defines the reference profile $\mathbf{r}_{m+1}(n)$ for the alignment of the $(m+1)$ th range profile $\mathbf{p}_{m+1}(n)$ as the previously aligned range profile, that is, $\mathbf{r}_{m+1}(n)=$ $\overline{\mathbf{p}}_{m}(n)$, according to the nomenclature used in Section 2. In Figure 12(a), some misalignment error accumulates, as clearly shown if we concentrate, for example, on the range history for the scatterers on sailboat stern. These range variations are not observed in Figure 7(a).

The error accumulation effect in Figure 12(a) has minor incidence on the results of Figure 7(a). Hence, the proposed range-bin alignment method based on the use of reference profiles is robust against this phenomenon.

On the other hand, Figure 12(b) shows the ISAR image obtained from the range profiles in Figure 12(a). This image is defocused in comparison with the ISAR image in
TABLE 4: Focusing indicators for the simulated ISAR images in Figures 7(b) and 12(b).

\begin{tabular}{lcc}
\hline & Entropy & Contrast \\
\hline Figure 7(b) & 7.17 & 10.60 \\
Figure 12(b) & 7.81 & 8.97 \\
\hline
\end{tabular}

Figure 7(b). This is obvious for the scatterers on the sailboat deck, for example. In fact, the same may be concluded if we calculate the focusing indicators for Figures 7(b) and 12(b), as Table 4 details.

\section{Comparison with State-of-the-Art Methods}

In previous section, we exposed the properties of the proposed alignment method by using both simulated and real data. Here, it is our intention to compare the proposed approach with state-of-the-art methods recently proposed in the literature: concretely, the global range alignment algorithm [20] and the minimum entropy-based approach [21].

The performance of the proposed subinteger range-bin alignment technique is similar to the one for these stateof-the-art methods, as shown next. However, the proposed approach can deal with extreme situations with large-range shifts from range profile to range profile. Moreover, its subinteger alignment capability is also noticeable and, unlike the other methods, the careful design of the optimization stage increases its robustness against possible convergence to local maxima.

To make the pertinent comparisons, we use the live data described in the following. Figure 13 shows the photo of a vessel illuminated by the millimeter-wave LFMCW radar prototype [26]. The radar parameters for this acquisition are detailed in Table 5.

Figures 14(a) and 14(b), respectively, show the range profiles and the ISAR image for this live acquisition without compensating the translational motion. The ISAR image 


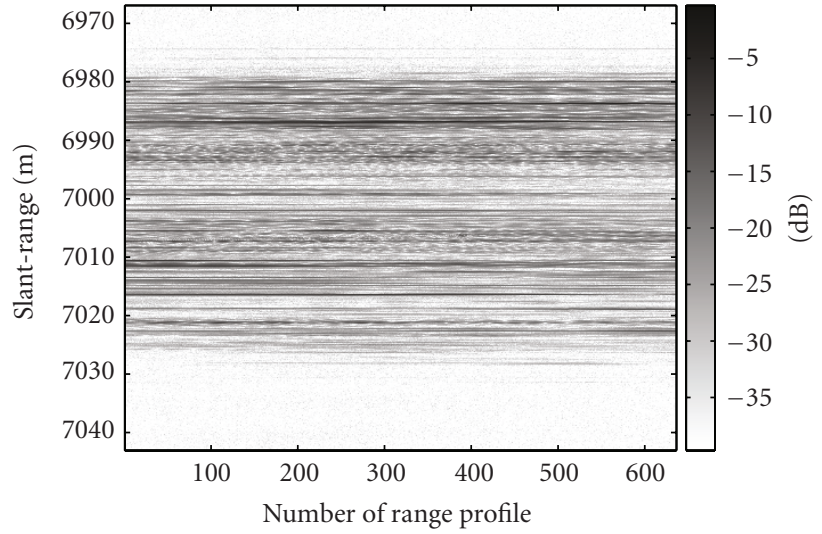

(a)

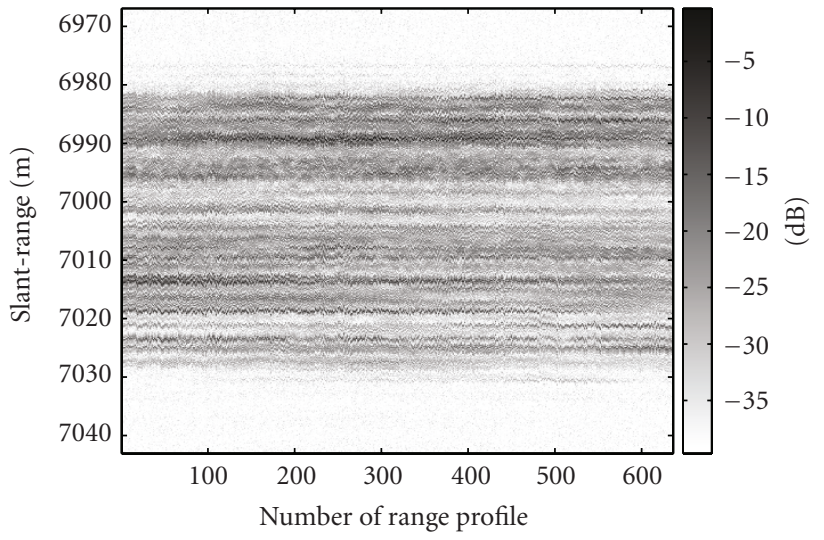

(b)

FIGURE 18: Range profiles aligned after applying (a) the proposed subinteger range-bin alignment method and (b) the global range alignment algorithm to the vessel data with artificially induced vibration.

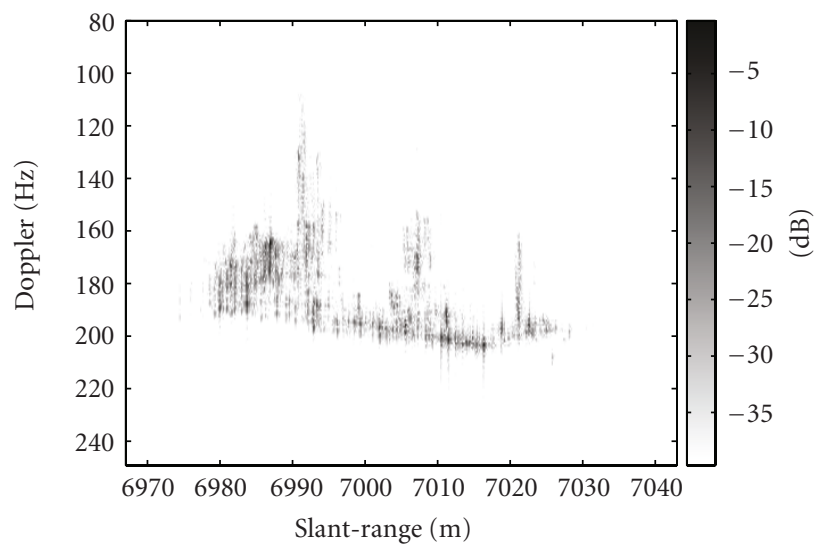

(a)

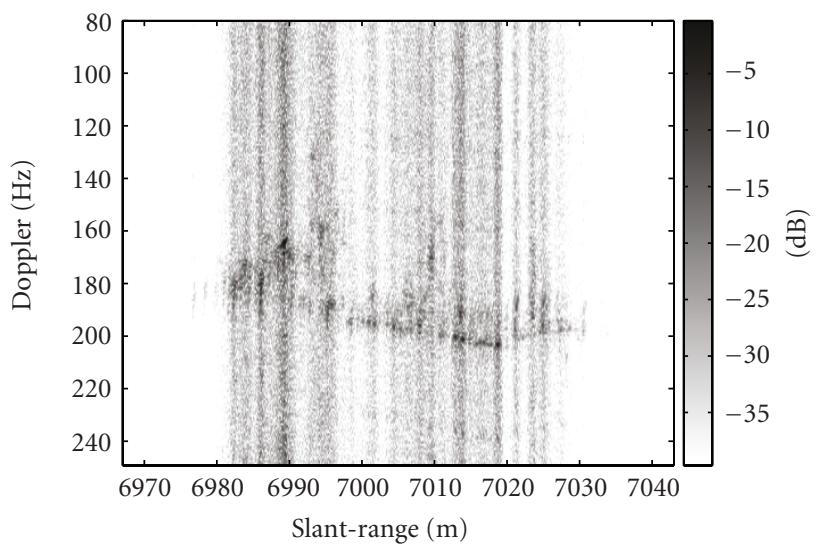

(b)

FIGURE 19: ISAR images obtained after applying (a) the proposed subinteger range-bin alignment method and (b) the global range alignment algorithm to the vessel data with artificially induced vibration.

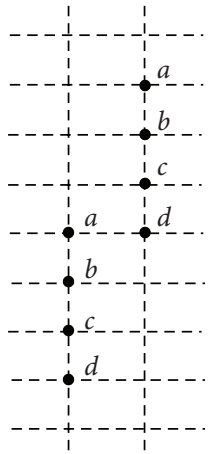

(a)

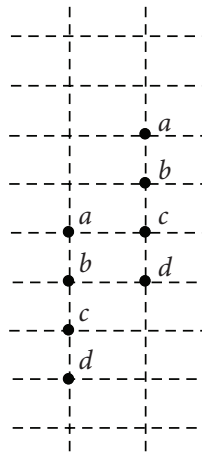

(b)

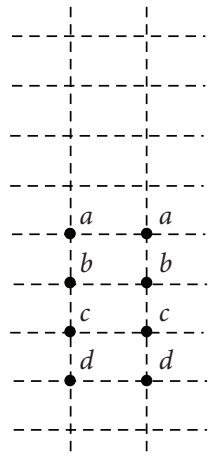

(c)
FIgURe 20: Alignment process of two range profiles for the global range alignment algorithm.

is largely blurred because of the radial component of the translational motion, which can easily be guessed from the leaning observed in the range profiles of Figure 14(a).
TABLE 5: Real radar parameters for the acquired data corresponding to the vessel in Figure 13.

\begin{tabular}{lc}
\hline Central frequency $\left(f_{0}\right)$ & $28.5 \mathrm{GHz}$ \\
Bandwidth $(\Delta f)$ & $1 \mathrm{GHz}$ \\
Ramp repetition frequency (PRF) & $500 \mathrm{~Hz}$ \\
$T_{C}$ & $0.2 \mathrm{~ms}$ \\
Illumination time $(\mathrm{CPI})$ & $1.27 \mathrm{~s}$ \\
\hline
\end{tabular}

Figure 15 presents the aligned range profiles and the focused ISAR image after applying the proposed subinteger range-bin alignment method and the method in [16] for the phase adjustment stage. Note the increase in the ISAR image quality. The masts and the deck appear focused. Certainly, the alignment produced by the proposed method seems to be good.

The focusing indicators, provided in Table 6, also speak about the quality enhancement after applying the subinteger range-bin alignment approach. 


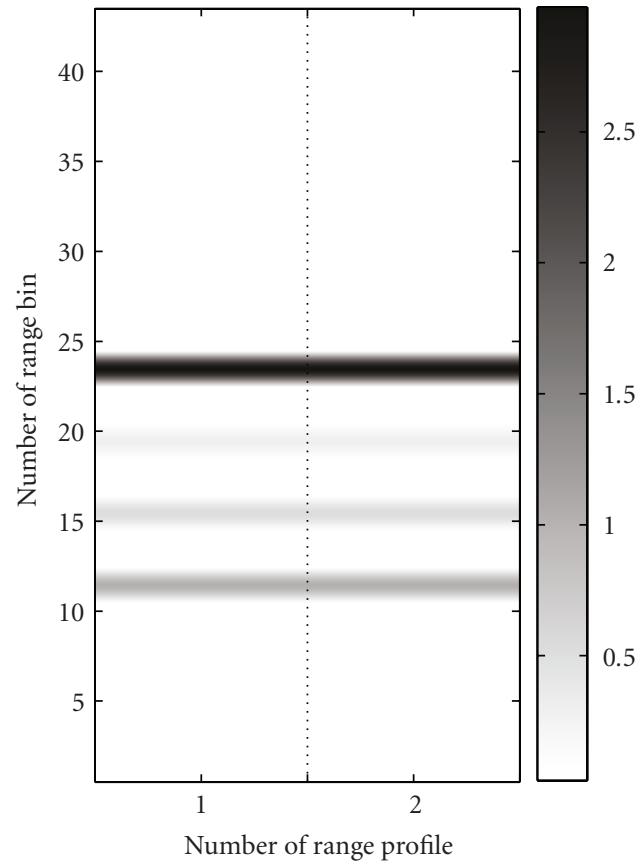

(a)

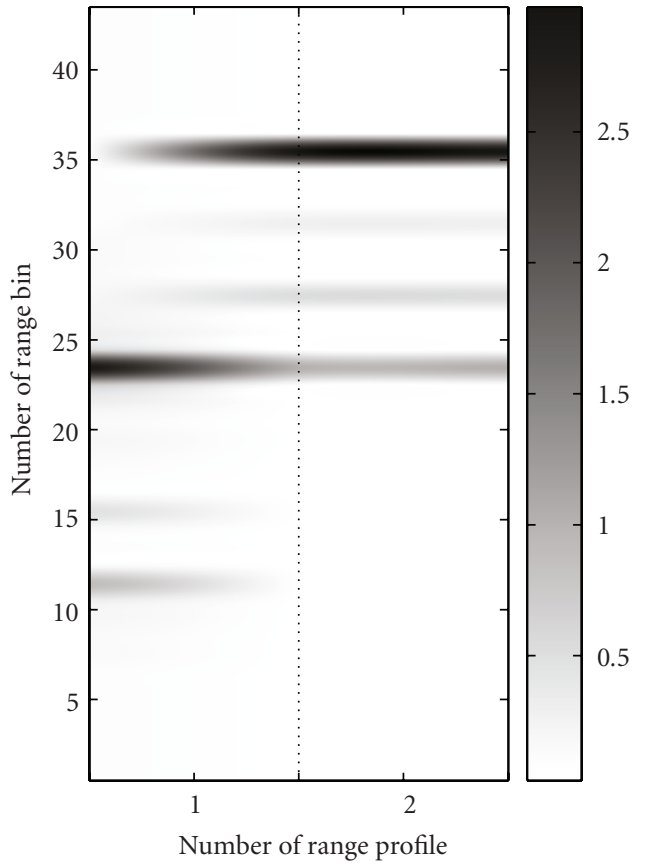

(b)

Figure 21: Alignment results for two simulated range profiles by applying (a) the proposed technique and (b) the global range alignment algorithm. The proposed method is robust against local maxima of its cost function. On the contrary, the global range alignment method may have convergence difficulties.

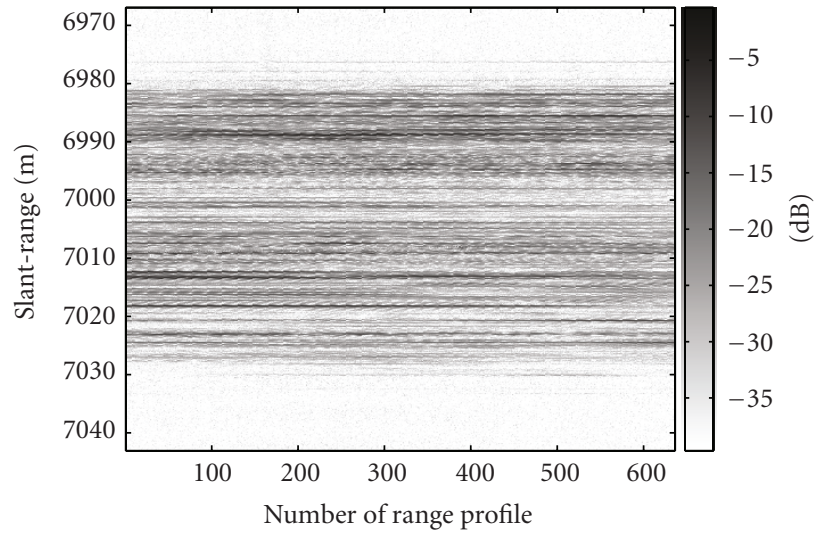

(a)

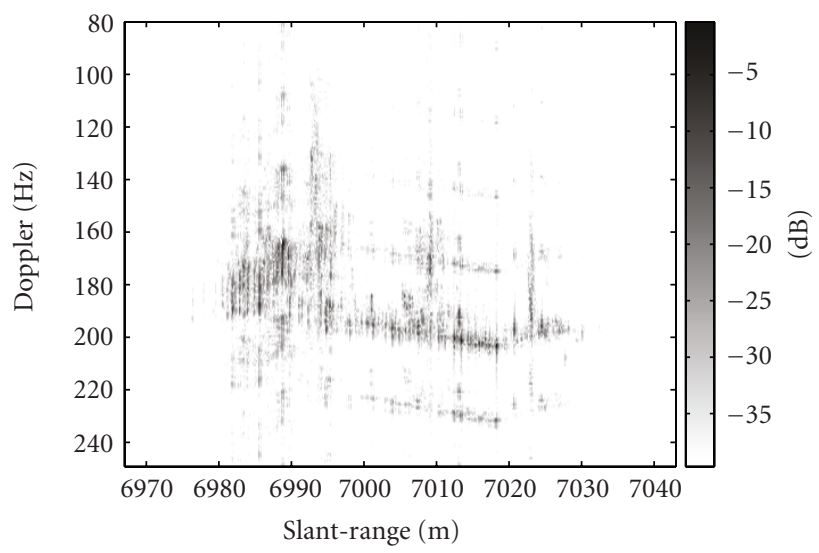

(b)

FIGURE 22: (a) Range profiles and (b) ISAR image for the real data of the vessel after using the minimum entropy-based approach.

TABLE 6: Focusing indicators for the real ISAR images of the vessel in Figures 14(b) and 15(b).

\begin{tabular}{lcc}
\hline & Entropy & Contrast \\
\hline Figure 14(b) & 9.32 & 8.7 \\
Figure 15(b) & 7.37 & 39.8 \\
\hline
\end{tabular}

5.1. Comparison with the Global Range Alignment Algorithm. The global range alignment method [20] is also a robust method which usually performs well in diverse scenarios. As a proof of that, Figures 16(a) and 16(b), respectively, show the range profiles and the ISAR image for the noncooperative data of Figure 14 after applying this global range alignment approach. Figure 16(b) is practically indistinguishable from the one obtained by the proposed approach (Figure 15(b)).

Hence, the global range alignment algorithm provides a good alignment. In fact, the focusing indicators for Figure 16(b) are almost the same as the ones for Figure 15(b). The entropy and contrast for Figure 16(b) are 7.36 and 39.9, respectively.

However, when we are faced with situations in which large range shifts from range profile to range profile may 


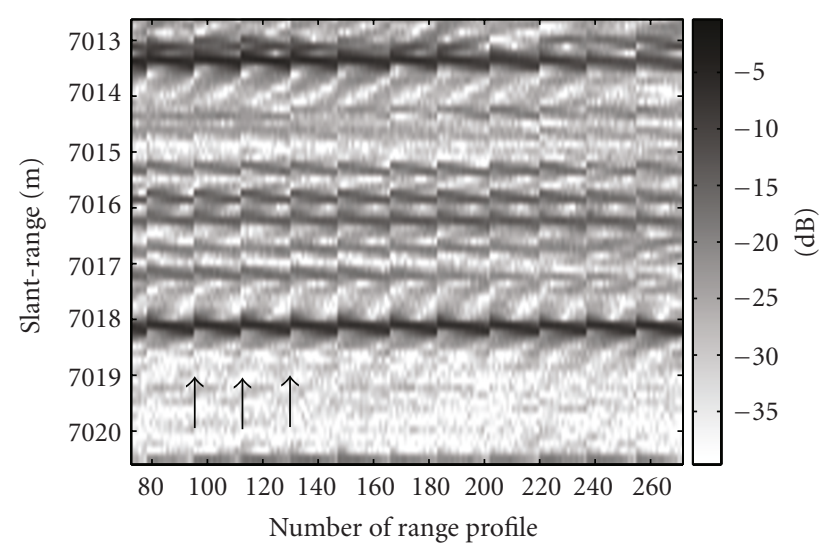

Figure 23: Zoom of Figure 22(a). The jumps in the range profiles are clearly visible.

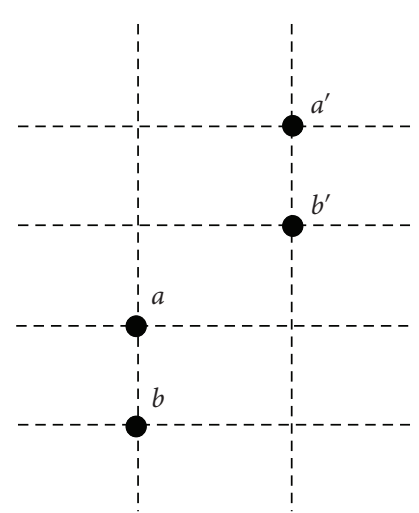

FIgURe 24: Two simple misaligned range profiles with little target scintillation.

arise, the global range alignment algorithm may fail, as also indicated in [21].

As a proof of that, let us concentrate on Figure 17, which shows the range profiles of the vessel with an artificially induced vibration. To simulate this vibration, each range profile has independently been shifted, with the shift being a Gaussian random variable of zero mean and a standard deviation of $10 \mathrm{~cm}$. This has let us simulate a large vibration.

Figure 18 presents the results provided by the proposed and the global range alignment methods for the range profiles shown in Figure 17. By analyzing Figure 18, we can conclude that the proposed technique is robust against large range displacements, whereas the global range alignment algorithm cannot properly solve these situations.

It is obvious that the poor performance of the global range alignment method shown in Figure 18(b) has influence on the subsequent obtained ISAR image. Figure 19 shows the ISAR images obtained after applying the proposed method and the global range alignment algorithm to the vessel data with artificially induced vibration. Again, the technique in [16] has been used for the phase adjustment step. Because of the misalignment, the ISAR image after the global range alignment approach is blurred. On the contrary, the ISAR image obtained with the proposed technique is very similar to the one given in Figure 15(b).

On the other hand, it is noticeable that the optimization stage in the proposed technique has carefully been designed. On the contrary, the global range alignment method may have difficulties with local maxima of its cost function $C^{\prime}$ [20, equation (8)]. To visualize this, let us consider two single misaligned range profiles, as Figure 20(a) indicates. The parameters $a, b, c$, and $d$ are the absolute values of the echoes in the corresponding range bins. An intermediate step in the optimization process of the global range alignment method is depicted in Figure 20(b). Figure 20(c) indicates the correct alignment of the two range profiles.

It can easily be shown that the values of $C^{\prime}$ in the situation depicted in Figures 20(a) and 20(b) are, respectively,

$$
\begin{gathered}
C_{(a)}^{\prime}=2\left(a^{2}+b^{2}+c^{2}+d^{2}+a d\right), \\
C_{(b)}^{\prime}=2\left(a^{2}+b^{2}+c^{2}+d^{2}+a c+d b\right) .
\end{gathered}
$$

From the optimization algorithm given in [20], if $C_{(a)}^{\prime}>$ $C_{(b)}^{\prime}$, that is, if $a d>a c+d b$, then the global range alignment algorithm will not converge to the correct result in Figure 20(c). Instead of that, it will try to refine the situation in Figure 20(a). As a proof of this fact, Figure 21 shows the two range profiles aligned after using the proposed method and the global range alignment algorithm, when $a=3, b=$ $0.25, c=0.5$, and $d=1$. The global range alignment method is clearly affected by the local maximum and does not converge to the situation in Figure 20(c). On the contrary, the proposed method can deal with these cases because of the careful design of its optimization stage.

5.2. Comparison with the Minimum Entropy-Based Approach. The minimum entropy-based approach [21] for range-bin alignment is an iterative approach which is based on integer shifts of the range profiles, unlike the proposed method and the global range alignment algorithm.

Figure 22(a) shows the range profiles obtained after applying the entropy-based method to the real data of the vessel in Figure 14. Figure 22(b) shows the corresponding ISAR image.

Apparently, the range profiles shown in Figure 22(a) seem to be properly aligned. However, the fact that the range profiles may only be shifted in integer steps implies that undesired jumps occur in the range history of the target scatterers. Figure 23 presents a zoom of Figure 22(a) which allows distinguishing the commented jumps. Some of them are marked with an arrow.

These discontinuities appearing in the range profiles induce an amplitude modulation in Doppler. This is the reason why spurious images are clearly visible in Figure 22(b). Hence, the ISAR image obtained with the minimum entropybased approach has a quality poorer than the one in Figure 15(b). The entropy and contrast for the ISAR image in Figure 22(b) (8.01 and 35.3, resp.) are also indicative of this quality decrease. Please refer to Table 6.

We have tried to implement an extension of the minimum entropy-based method in order to consider subinteger 


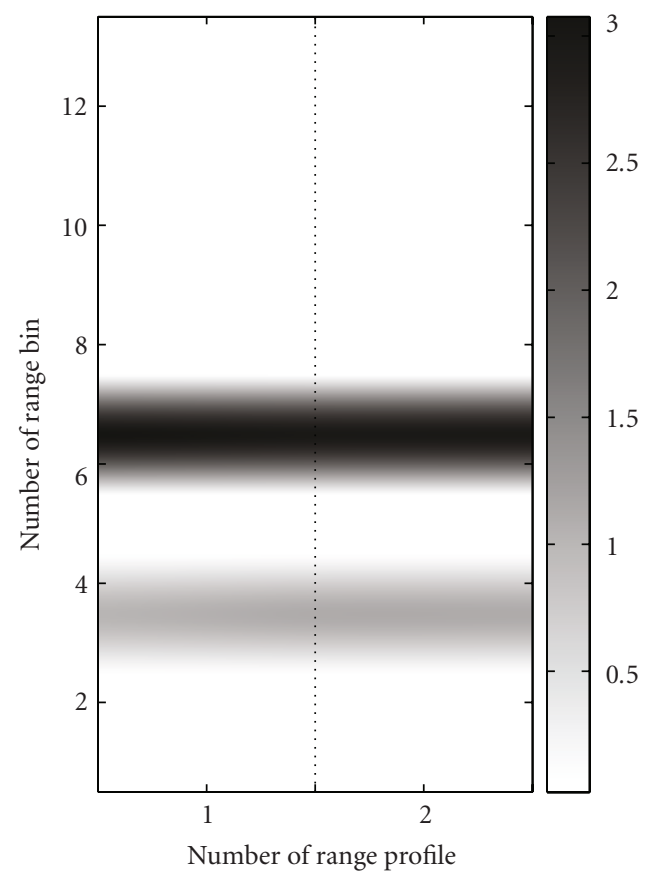

(a)

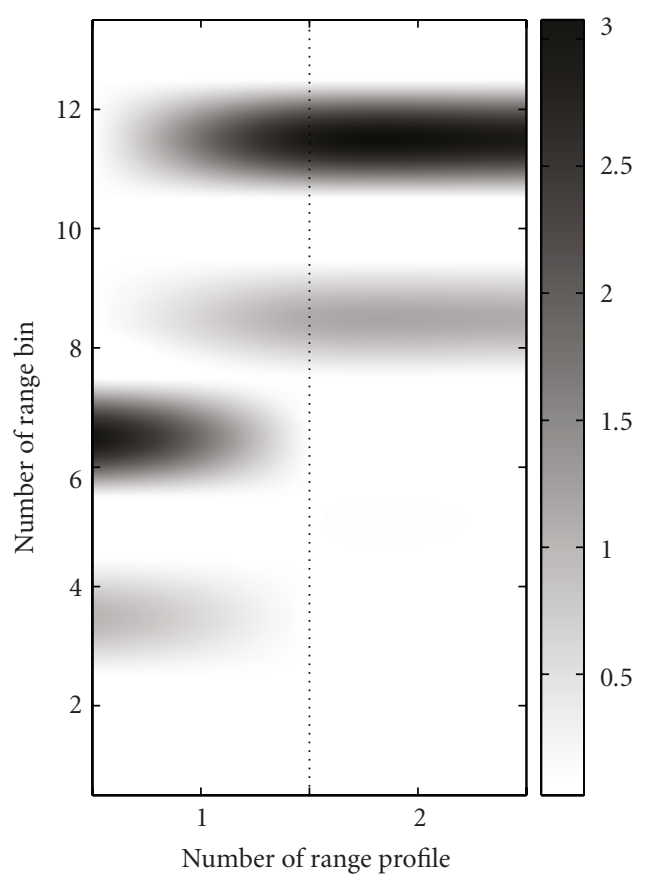

(b)

Figure 25: Alignment results for the two simulated range profiles in Figure 24 by applying (a) the proposed technique and (b) the minimum entropy-based approach.

range-bin alignments. This has been done in a way similar to the optimization stage given in the proposed method. Unfortunately, the commented jumps still appear when applying this extension.

On the other hand, the minimum entropy-based approach may also have problems with local maxima. As a proof of that, let us consider two misaligned range profiles as shown in Figure 24. The values $a, a^{\prime}, b$, and $b^{\prime}$ are the absolute values of the echoes in the corresponding range bins. Let us also assume that we have a little target scintillation, in such a way that we assume $a \approx a^{\prime}$ and $b \approx b^{\prime}$.

From the equations given in [21], it can easily be shown that the minimum entropy-based method will not be able to align the two range profiles in Figure 24, if the next two conditions are met:

$$
\begin{gathered}
a \ln a+b \ln b>a \ln a^{\prime}+b \ln b^{\prime}, \\
a^{\prime} \ln a^{\prime}+b^{\prime} \ln b^{\prime}>a^{\prime} \ln a+b^{\prime} \ln b .
\end{gathered}
$$

Let us consider that $a=3.1, b=1, a^{\prime}=3$, and $b^{\prime}=1.1$. These values satisfy (13). Figure 25(b) shows the two range profiles after applying the minimum entropy-based approach for this case. As predicted, the method is unable to align the two range profiles. On the contrary, the proposed method can align the two range profiles, as shown in Figure 25(a). Again, we would like to highlight that the optimization stage of the proposed method has carefully been designed.

\section{Conclusions}

The traditional imaging sensors, such as cameras or laser radars, may have a reduced performance in adverse meteorological conditions or in difficult scenarios where, for example, smoke screens are present. ISAR is an all-weather radar technique which may provide images of noncooperative targets in such adverse environments. Hence, such images are interesting for defense and security applications. Furthermore, the ISAR images may be exploited for subsequent recognition/identification tasks.

Unfortunately, the standard ISAR images are usually blurred because of the target motion. Motion compensation techniques should be applied in order to have focused ISAR images. Generally, it is at least necessary to compensate the radial component of the translational motion. To achieve this, the methods for translational motion compensation work in two stages: range-bin alignment and phase adjustment.

In order to increase the quality of the ISAR images, the range-bin alignment step must properly be designed. In this paper, we have proposed a range-bin alignment method based on the envelope correlation between the range profiles and their corresponding reference profiles, calculated as a combination of the previously aligned range profiles. Furthermore, the method achieves an accurate subinteger refinement for the range profile alignment. This subinteger adjustment is based on an optimization stage which has carefully been designed in order to avoid convergence to undesired local maxima. 
The paper addresses the performance of the proposed algorithm in an exhaustive manner, by using both simulated and real data from LFMCW radars. In this context, it has been shown that the method is robust against target scintillation, noise, and clutter. Its robustness against the error accumulation effect has also been verified.

On the other hand, the proposed method has also been compared with recently proposed state-of-the-art range-bin alignment methods, such as the global range alignment algorithm and the minimum entropy-based approach. We have verified that the subinteger feature of the proposed method provides us with extremely accurate range-bin alignments, in contrast to the minimum entropy-based approach. It has also been shown that the method may deal with large range shifts from range profile to range profile, unlike the global range alignment algorithm. Finally, the careful design of the proposed optimization stage has been highlighted. We have addressed simple simulated examples in which both the global range alignment algorithm and the minimum entropy-based technique have problems with local maxima.

The proposed algorithm is robust in many scenarios and is hence a very interesting alternative for the range-bin alignment stage in the task of ISAR translational motion compensation. The improved obtained ISAR images may be of interest for subsequent automatic target recognition methods.

\section{Acknowledgments}

This work was financially supported by the Spanish National Board of Scientific and Technology Research under Project TEC2008-02148/TEC. The authors thank Dr. A. Blanco-delCampo, Dr. A. Asensio-López, and Dr. B. P. Dorta-Naranjo for providing the live data of the sailboat and the vessel.

\section{References}

[1] S. A. Hovanessian, Introduction to Sensor Systems, Artech House, Boston, Mass, USA, 1988.

[2] A. V. Jelalian, Laser Radar Systems, Artech House, Boston, Mass, USA, 1992.

[3] G. R. Osche and D. S. Young, "Imaging laser radar in the near and far infrared," Proceedings of the IEEE, vol. 84, no. 2, pp. 103-125, 1996.

[4] H.-Y. Chen, I.-Y. Tarn, and Y.-J. Hwang, "Infrared extinction of the powder of brass $70 \mathrm{Cu} / 30 \mathrm{Zn}$ calculated by the FDTD and turning bands methods," IEEE Transactions on Geoscience and Remote Sensing, vol. 33, no. 6, pp. 1321-1324, 1995.

[5] C.-C. Chen and H. C. Andrews, "Target motion induced radar imaging," IEEE Transactions on Aerospace and Electronic Systems, vol. 16, no. 1, pp. 2-14, 1980.

[6] D. A. Ausherman, A. Kozma, J. L. Walker, H. M. Jones, and E. C. Poggio, "Developments in radar imaging," IEEE Transactions on Aerospace and Electronic Systems, vol. 20, no. 4, pp. 363-400, 1984.

[7] K.-T. Kim, D.-K. Seo, and H.-T. Kim, "Efficient classification of ISAR images," IEEE Transactions on Antennas and Propagation, vol. 53, no. 5, pp. 1611-1621, 2005.
[8] B. K. Shreyamsha Kumar, B. Prabhakar, K. Suryanarayana, V. Thilagavathi, and R. Rajagopal, "Target identification using harmonic wavelet based ISAR imaging," EURASIP Journal on Applied Signal Processing, vol. 2006, Article ID 86053, 13 pages, 2006.

[9] E. Radoi, A. Quinquis, and F. Totir, "Supervised selforganizing classification of superresolution ISAR images: an anechoic chamber experiment," EURASIP Journal on Applied Signal Processing, vol. 2006, Article ID 35043, 14 pages, 2006.

[10] S. Musman, D. Kerr, and C. Bachmann, "Automatic recognition of ISAR ship images," IEEE Transactions on Aerospace and Electronic Systems, vol. 32, no. 4, pp. 1392-1404, 1996.

[11] M. I. Skolnik, Introduction to Radar Systems, McGraw Hill Higher Education, McGraw Hill, New York, NY, USA, 3rd edition, 2001.

[12] D. R. Wehner, High Resolution Radar, Artech House, Boston, Mass, USA, 2nd edition, 1995.

[13] V. C. Chen and W. J. Miceli, "Simulation of ISAR imaging of moving targets," IEE Proceedings: Radar, Sonar and Navigation, vol. 148, no. 3, pp. 160-166, 2001.

[14] V. C. Chen, Time-Frequency Transforms for Radar Imaging and Signal Analysis, Artech House, Boston, Mass, USA, 2002.

[15] J. L. Walker, "Range-Doppler imaging of rotating objects," IEEE Transactions on Aerospace and Electronic Systems, vol. 16, no. 1, pp. 23-52, 1980.

[16] L. I. Xi, G. Liu, and N. Jinlin, "Autofocusing of ISAR images based on entropy minimization," IEEE Transactions on Aerospace and Electronic Systems, vol. 35, no. 4, pp. 1240-1252, 1999.

[17] J. M. Muñoz-Ferreras, J. Calvo-Gallego, F. Pérez-Martínez, A. Blanco-del-Campo, A. Asensio-López, and B. P. DortaNaranjo, "Motion compensation for ISAR based on the shift-and-convolution algorithm," in Proceedings of the IEEE Conference on Radar, pp. 366-370, Verona, NY, USA, April 2006.

[18] G. Y. Delisle and H. Wu, "Moving target imaging and trajectory computation using ISAR," IEEE Transactions on Aerospace and Electronic Systems, vol. 30, no. 3, pp. 887-899, 1994.

[19] J. M. Muñoz-Ferreras and F. Pérez-Martínez, "Extended envelope correlation for range bin alignment in ISAR," in Proceedings of the IET International Conference on Radar Systems, pp. 65-68, Edinburg, UK, October 2007.

[20] J. Wang and D. Kasilingam, "Global range alignment for ISAR," IEEE Transactions on Aerospace and Electronic Systems, vol. 39, no. 1, pp. 351-357, 2003.

[21] D. Zhu, L. Wang, Y. Yu, Q. Tao, and Z. Zhu, "Robust ISAR range alignment via minimizing the entropy of the average range profile," IEEE Geoscience and Remote Sensing Letters, vol. 6, no. 2, pp. 204-208, 2009.

[22] B. D. Steinberg, "Microwave imaging of aircraft," Proceedings of the IEEE, vol. 76, no. 12, pp. 1578-1592, 1988.

[23] D. E. Wahl, P. H. Eichel, D. C. Ghiglia, and C. V. Jakowatz Jr., "Phase gradient autofocus - a robust tool for high resolution SAR phase correction," IEEE Transactions on Aerospace and Electronic Systems, vol. 30, no. 3, pp. 827-835, 1994.

[24] M. Martorella, F. Berizzi, and B. Haywood, "Contrast maximisation based technique for 2-D ISAR autofocusing," IEE Proceedings: Radar, Sonar and Navigation, vol. 152, no. 4, pp. 253-262, 2005. 
[25] J. A. Nelder and R. Mead, "A simplex method for function minimization," The Computer Journal, vol. 7, pp. 308-313, 1965.

[26] A. Blanco-del-Campo, A. Asensio-López, B. P. Dorta-Naranjo, et al., "Millimeter-wave radar demonstrator for high resolution imaging," in Proceedings of the 1st European Radar Conference (EuRAD '04), pp. 65-68, Amsterdam, The Netherlands, October 2004. 